\title{
Minimal Positive Stencils in Meshfree Finite Difference Methods for the Poisson Equation
}

\author{
Benjamin Seibold \\ Department of Mathematics \\ Massachusetts Institute of Technology \\ 77 Massachusetts Avenue \\ Cambridge MA 02139, USA
}

February 11, 2013

\begin{abstract}
Meshfree finite difference methods for the Poisson equation approximate the Laplace operator on a point cloud. Desirable are positive stencils, i.e. all neighbor entries are of the same sign. Classical least squares approaches yield large stencils that are in general not positive. We present an approach that yields stencils of minimal size, which are positive. We provide conditions on the point cloud geometry, so that positive stencils always exist. The new discretization method is compared to least squares approaches in terms of accuracy and computational performance.
\end{abstract}

\section{Introduction}

The numerical approximation of the Poisson equation is a fundamental task encountered in many applications. Often it appears as a subproblem in a more complex computation, for instance as a projection step in the simulation of incompressible flows [1. Finite difference methods approximate the equation on a finite number of points. If the points can be placed on a regular grid, the approximation is simple and yields symmetric matrices. However, in many cases a regular point distribution is not possible or desired. Examples are the explicit representation of complex geometries, or the point positions may be given by the application, for instance from scattered measurements or in particle methods [8]. If the points are distributed irregularly, neighborhood relations have to be established. This could be done by 
constructing a mesh. However, meshing can be costly, and thus may not be desired in applications with time-dependent geometries. Instead, meshfree neighborhood criteria can be defined, and meshfree finite difference stencils constructed. Consistency conditions for stencils are derived in Sect. 2, We are interested in approaches that yield M-matrices, as explained in Sect. 3. This requires the stencils to be positive. Least squares approaches, outlined in Sect. 4, in general fail to yield positive stencils. In Sect. 5]we present a new approach, based on sign constrained linear minimization, that yields positive stencils. In Sect. 6] we provide conditions on the point cloud geometry, so that positive stencils are guaranteed to exist. Further conditions, derived in Sect. 7, ensure an M-matrix structure. In Sect. 8 the new approach is compared to classical methods by numerical experiments.

\section{Meshfree Finite Differences for the Poisson Equa- tion}

Consider the Poisson equation to be solved inside a domain $\Omega \subset \mathbb{R}^{d}$

$$
\begin{cases}-\Delta u=f & \text { in } \Omega \\ u=g & \text { on } \Gamma_{D} \\ \frac{\partial u}{\partial n}=h & \text { on } \Gamma_{N}\end{cases}
$$

where $\Gamma_{D} \cup \Gamma_{N}=\partial \Omega$. Let a point cloud $X=\left\{\boldsymbol{x}_{1}, \ldots, \boldsymbol{x}_{n}\right\} \subset \bar{\Omega}$ be given, which consists of interior points $X_{i} \subset \Omega$ and boundary points $X_{b} \subset \partial \Omega$. The point cloud is meshfree, i.e. no information about connection of points is provided. Meshfree finite difference approaches convert problem (11) into a linear system

$$
A \cdot \hat{\boldsymbol{u}}=\hat{\boldsymbol{f}},
$$

where the vector $\hat{\boldsymbol{u}}$ contains approximations to the values $u\left(\boldsymbol{x}_{i}\right)$. The $i$-th row of the matrix $A$ consists of the stencil corresponding to the point $\boldsymbol{x}_{i}$. We assume that (11) admits a unique smooth solution.

\subsection{Consistent Derivative Approximation}

Consider a function $u \in C^{2}\left(\Omega \subset \mathbb{R}^{d}, \mathbb{R}\right)$. We wish to approximate $\Delta u\left(\boldsymbol{x}_{0}\right)$ using the function values of a finite number of points in a circular neighborhood $\left(\boldsymbol{x}_{0}, \boldsymbol{x}_{1}, \ldots, \boldsymbol{x}_{m}\right) \in B\left(\boldsymbol{x}_{0}, r\right)$, where $B\left(\boldsymbol{x}_{0}, r\right)=\left\{\boldsymbol{x} \in \bar{\Omega}:\left\|\boldsymbol{x}-\boldsymbol{x}_{0}\right\|<r\right\}$. Define the distance vectors $\overline{\boldsymbol{x}}_{i}=\boldsymbol{x}_{i}-\boldsymbol{x}_{0} \forall i=0, \ldots, m$. The function value 
at each neighboring point $u\left(\boldsymbol{x}_{i}\right)$ can be expressed by a Taylor expansion

$$
u\left(\boldsymbol{x}_{i}\right)=u\left(\boldsymbol{x}_{0}\right)+\nabla u\left(\boldsymbol{x}_{0}\right) \cdot \overline{\boldsymbol{x}}_{i}+\frac{1}{2} \nabla^{2} u\left(\boldsymbol{x}_{0}\right):\left(\overline{\boldsymbol{x}}_{i} \cdot \overline{\boldsymbol{x}}_{i}^{T}\right)+e_{i} .
$$

We use the matrix scalar product $A: B=\sum_{i, j} A_{i j} B_{i j}$. The error in the expansion is of order $e_{i}=O\left(r^{3}\right)$. A linear combination with coefficients $\left(s_{0}, \ldots, s_{m}\right)$ equals

$$
\begin{array}{r}
\sum_{i=0}^{m} s_{i} u\left(\boldsymbol{x}_{i}\right)=u\left(\boldsymbol{x}_{0}\right)\left(\sum_{i=0}^{m} s_{i}\right)+\nabla u\left(\boldsymbol{x}_{0}\right) \cdot\left(\sum_{i=1}^{m} s_{i} \overline{\boldsymbol{x}}_{i}\right) \\
+\nabla^{2} u\left(\boldsymbol{x}_{0}\right):\left(\frac{1}{2} \sum_{i=1}^{m} s_{i}\left(\overline{\boldsymbol{x}}_{i} \cdot \overline{\boldsymbol{x}}_{i}^{T}\right)\right)+\left(\sum_{i=1}^{m} s_{i} e_{i}\right) .
\end{array}
$$

This approximates the Laplacian, i.e. $\sum_{i=0}^{m} s_{i} u\left(\boldsymbol{x}_{i}\right)=\Delta u\left(\boldsymbol{x}_{0}\right)+O\left(r^{3}\right)$, if exactness for constant, linear and quadratic functions is satisfied

$$
\sum_{i=0}^{m} s_{i}=0 \quad, \quad \sum_{i=1}^{m} \overline{\boldsymbol{x}}_{i} s_{i}=0 \quad, \quad \sum_{i=1}^{m}\left(\overline{\boldsymbol{x}}_{i} \cdot \overline{\boldsymbol{x}}_{i}^{T}\right) s_{i}=2 I .
$$

Definition 1. A stencil $\left(s_{0}, \ldots, s_{m}\right)$ to a set of points $\left(\boldsymbol{x}_{0}, \boldsymbol{x}_{1}, \ldots, \boldsymbol{x}_{m}\right) \in$ $B\left(\boldsymbol{x}_{0}, r\right)$ is called consistent (with the Laplace operator), if the constraints (3) are satisfied.

The linear and quadratic constraints in (3) can be formulated as a linear system of equations

$$
V \cdot s=b,
$$

where $V \in \mathbb{R}^{k \times m}$ is the Vandermonde matrix given by $\overline{\boldsymbol{x}}_{1}, \ldots, \overline{\boldsymbol{x}}_{m}$, and $s \in \mathbb{R}^{m}$ is the stencil vector. In $2 \mathrm{~d}$, with $\overline{\boldsymbol{x}}_{i}=\left(\bar{x}_{i}, \bar{y}_{i}\right)$, the system reads as

$$
V=\left(\begin{array}{ccc}
\bar{x}_{1} & \ldots & \bar{x}_{m} \\
\bar{y}_{1} & \ldots & \bar{y}_{m} \\
\bar{x}_{1} \bar{y}_{1} & \ldots & \bar{x}_{m} \bar{y}_{m} \\
\bar{x}_{1}^{2} & \ldots & \bar{x}_{m}^{2} \\
\bar{y}_{1}^{2} & \ldots & \bar{y}_{m}^{2}
\end{array}\right) \quad, \quad \boldsymbol{b}=\left(\begin{array}{c}
0 \\
0 \\
0 \\
2 \\
2
\end{array}\right) .
$$

The number of constraints is $k=\frac{d(d+3)}{2}$. The constant constraint in (3) yields $s_{0}=-\sum_{i=1}^{m} s_{i}$. Neumann boundary points can be treated in a similar manner. Approximating $\frac{\partial u}{\partial \boldsymbol{n}}\left(\boldsymbol{x}_{0}\right)$ by $\sum_{i=0}^{m} s_{i} u\left(\boldsymbol{x}_{i}\right)$ leads to the constraints

$$
\sum_{i=0}^{m} s_{i}=0 \quad, \quad \sum_{i=1}^{m} \overline{\boldsymbol{x}}_{i} s_{i}=\boldsymbol{n} .
$$


For each point, a meshfree finite difference approximation consists of two steps: First, define which points are its neighbors. Typically, more neighbors than constraints are chosen. Second, select a stencil. If (3) is underdetermined, a minimization problem is formulated to select a unique stencil.

A set of neighbors around a central point is called in general configuration, if the Vandermonde matrix $V$ has full rank. For $m$ neighboring points, one has no solution if $m<k$, infinitely many solutions if $m>k$, and one solution if $m=k$. In this case, the stencil $s=V^{-1} \cdot \boldsymbol{b}$ can be computed by elimination, or by formulas for the determinant of a multivariate Vandermonde matrix [12]. If the points are not in general configuration, e.g. for regular grids, the above rules may fail. A solution can exist for $m<k$ (e.g. 5-point stencil in 2d), and no solution may exist for $m>k$ (see example in [15, p. 59]). The concept of general configuration is unhandy and too strict. Solutions may be acceptable, even if $V$ does not have full rank. The geometric condition presented in Sect. [6 ensures the existence of stencils.

\subsection{Minimal and Positive Stencils}

Definition 2. A consistent stencil $\left(s_{0}, \ldots, s_{m}\right)$ is called minimal, if $m \leq k$.

Minimal stencils are beneficial for the sparsity of the system matrix, resulting in a lower memory consumption and a faster solution of system (2). The total number of neighboring points is proportional to the effort of applying the matrix to a vector, which is proportional to the time for one step of a (semi-)iterative linear solver. Of course, the few neighbors have to be chosen wisely, to preserve good convergence rates of iterative solvers. The results in [16, 17] indicate that this is the case with the presented approach.

Remark 1. For minimal stencils, it is impossible that the stencil values depend continuously on the point positions. Consider six points around a central point (in $2 \mathrm{~d}$ ), five of which are selected neighbors. Consider a continuous movement of one of the neighbors and the sixth point, such that at the end these two points have swapped their positions, without them ever being in the same place. At some instance during this movement, the sixth point has to become a neighbor, resulting in a jump in the stencil values.

Remark 2. If used in a particle method, the lack of smoothness in minimal stencils (Rem. 1) may lead to a non-conservative scheme. In an isolated Poisson solver and in particle methods that are not conservative by construction (such as the finite pointset method [8]) the advantage of optimal sparsity often outweighs this drawback. 
Definition 3. A consistent stencil $\left(s_{0}, \ldots, s_{m}\right)$ is called positive, if $s_{1}, \ldots, s_{m} \geq 0$. Due to (3) and (6), this implies for the central point $s_{0}<0$.

Positive stencils yields the system matrix in (2) to be an L-matrix (Def. 4), which gives rise to an M-matrix structure (see Sect. 3). The desirability of positive stencils has been pointed out by Demkowicz, Karafiat and Liszka [2]. Classical approaches do in general not yield positive stencils. An "optimal star selection" 3] makes positive stencils likely, but they are not guaranteed (see Fig. 5). Fürst and Sonar derive topological conditions on point clouds for positive least squares stencils in 1d [6]. In Sect. 5 we present a strategy that approximates the Poisson equation (1) on a point cloud by minimal positive stencils. In Sect. 6 conditions on a point cloud are presented (in $2 \mathrm{~d}$ and $3 \mathrm{~d}$ ) which guarantee the existence of positive stencils.

\section{M-Matrices}

Meshfree finite difference matrices are in general non-symmetric. Consider two points $\boldsymbol{x}_{i}$ and $\boldsymbol{x}_{j}$, each being a neighbor of the other, and a third point $\boldsymbol{x}_{k}$ which is a neighbor of $\boldsymbol{x}_{i}$ but not a neighbor of $\boldsymbol{x}_{j}$. Since each stencil entry depends on all its neighbors, the point $\boldsymbol{x}_{k}$ influences the matrix entry $a_{i j}$, but not the matrix entry $a_{j i}$.

The negative Laplace operator in (1) is positive definite. For nonsymmetric matrices, we have to ask for slightly less than positive definiteness. A property which implies a maximum principle and the convergence of linear solvers, is the M-matrix structure.

Definition 4. A square matrix $A=\left(a_{i j}\right)_{i j} \in \mathbb{R}^{n \times n}$ is called Z-matrix, if $a_{i j} \leq 0 \forall i \neq j$. A Z-matrix is called $L$-matrix, if $a_{i i}>0 \forall i$.

We write $A \geq 0$ for $a_{i j} \geq 0 \forall i, j$. The same notation applies to vectors.

Definition 5. A regular matrix $A$ is called inverse positive, if $A^{-1} \geq 0$.

Definition 6. A Z-matrix is called M-matrix, if it is inverse positive.

We use the M-matrix property, since it yields a sufficient condition for inverse positivity. There are inverse positive matrices that are not M-matrices, so another approach would be to employ alternative characterizations of inverse positive matrices [5]. 


\subsection{Benefits of an M-Matrix Structure}

The Poisson equation satisfies maximum principles. For instance, consider (11) with Dirichlet boundary conditions only. If $f \leq 0$ and $g \leq 0$, then the solution satisfies $u \leq 0$ [4]. A discretization by an M-matrix mimics this property in a discrete maximum principle.

Theorem 1. Let $A$ be an M-matrix. Then $A \boldsymbol{x} \leq 0$ implies $\boldsymbol{x} \leq 0$. Conversely, a Z-matrix satisfying $A \boldsymbol{x} \leq 0 \Rightarrow \boldsymbol{x} \leq 0$ is an M-matrix.

Proof. A is an M-matrix, thus $A^{-1} \geq 0$ by definition. Let $\boldsymbol{y}=A \boldsymbol{x}$. Then $\boldsymbol{x}=A^{-1} \boldsymbol{y}$. The component-wise inequalities $A^{-1} \geq 0$ and $\boldsymbol{y} \leq 0$ imply $\boldsymbol{x} \leq 0$. The reverse statement is proved in [13, p. 29].

Theorem 2. If $A$ is an M-matrix, and $D$ its diagonal part, then $\rho(I-$ $\left.D^{-1} A\right)<1$, thus the Jacobi and the Gauß-Seidel iteration converge.

Proof. The convergence of the Jacobi iteration is given in [7]. The GaußSeidel convergence follows from the Stein-Rosenberg-Theorem [20].

The performance of multigrid methods for meshfree finite difference matrices has been investigated in [16, 17]. Further benefits of an M-matrix structure with respect to linear solvers can be found in [20].

\subsection{A Sufficient Condition for an M-Matrix Structure}

Conditions that imply the M-matrix property are required, since the inverse matrix is typically not directly available. Let the unknowns be labeled by an index set $I$. We consider square matrices $A \in \mathbb{R}^{I \times I}$.

Definition 7. The graph $G(A)$ of a matrix $A$ is defined by $G(A)=\{(i, j) \in$ $\left.I \times I: a_{i j} \neq 0\right\}$. The index $i \in I$ is called connected to $j \in I$, if a chain $i=$ $i_{0}, i_{1}, \ldots, i_{k-1}, i_{k}=j \in I$ exists, such that $\left(i_{\nu-1}, i_{\nu}\right) \in G(A) \forall \nu=1, \ldots, k$.

Remark 3. For a finite difference matrix, each index $i \in I$ corresponds to a point $\boldsymbol{x}_{i}$. The index $i \in I$ being connected to $j \in I$ means that the point $\boldsymbol{x}_{i}$ connects (indirectly) to the point $\boldsymbol{x}_{j}$ via stencil entries.

Definition 8. A finite difference matrix is called essentially irreducible if every point is connected to a Dirichlet boundary point.

Remark 4. A finite difference matrix that is not essentially irreducible, is singular, since the points that are not connected to a Dirichlet point form a singular submatrix. 
Definition 9. A matrix $A \in \mathbb{R}^{I \times I}$ is called essentially diagonally dominant, if it is weakly diagonally dominant $\left(\forall i \in I:\left|a_{i i}\right| \geq \sum_{k \neq i}\left|a_{i k}\right|\right)$, and every point $i \in I$ is connected to a point $j \in I$ which satisfies the strict diagonal dominance relation $\left|a_{j j}\right|>\sum_{k \neq j}\left|a_{j k}\right|$.

Theorem 3. An L-matrix arising as a finite difference discretization of (11) is essentially diagonally dominant, if it is essentially irreducible.

Proof. For an L-matrix the constant relation in (3) implies the weak diagonal dominance relation for every interior and Neumann point. Each row corresponding to a Dirichlet point satisfies the strict diagonal dominance relation.

Theorem 4. An essentially diagonally dominant L-matrix is an M-matrix.

Proof. The proof is given in [7, p. 153].

If problem (11) can be discretized by positive stencils and every point is connected to a Dirichlet point, then the resulting matrix is an M-matrix.

\section{Least Squares Approaches}

Classical approaches for meshfree derivative approximation are moving least squares methods, based on scattered data interpolation [9], and local approximation methods, based on generalized finite difference methods [11. Their application to meshfree settings has been analyzed in [3, 10]. Differences between moving and local approaches have been investigated in [15].

Around a central point $\boldsymbol{x}_{0}$, points inside a radius $r$ are considered. A distance weight function $w(\delta)$ is defined, which is small for $\delta>r$. We consider interpolating 1 approaches with $w(\delta)=\delta^{-\alpha}$. Each neighboring point $\boldsymbol{x}_{i}$ is assigned a weight $w_{i}=w\left(\left\|\boldsymbol{x}_{i}-\boldsymbol{x}_{0}\right\|_{2}\right)$. A unique stencil is defined via a quadratic minimization problem

$$
\min \sum_{i=1}^{n} \frac{s_{i}^{2}}{w_{i}}, \text { s.t. } V \cdot s=\boldsymbol{b} .
$$

Using $W=\operatorname{diag}\left(w_{i}\right)$, its solution is

$$
\boldsymbol{s}=W V^{T}\left(V W V^{T}\right)^{-1} \cdot \boldsymbol{b} .
$$

\footnotetext{
${ }^{1}$ If $\lim _{\delta \rightarrow 0} w(\delta)$ exists, the approach is called approximating, otherwise interpolating.
} 
After the weights $w_{i}$ are evaluated, the $k \times k$ matrix $V W V^{T}$ has to be set up, the linear system $\left(V W V^{T}\right) \cdot \boldsymbol{v}=\boldsymbol{b}$ to be solved, and the product $s=W V^{T} \cdot \boldsymbol{v}$ to be computed. This requires $k(k+1) m+\frac{k^{3}}{3}$ floating point operations [15, p. 150]. Least squares approaches do not yield minimal stencils, unless exactly $k$ neighbors are considered. In general, they also do not yield positive stencils.

Example 1. Consider $\boldsymbol{x}_{0}=(0,0)$ and 6 neighbors on the unit circle $\boldsymbol{x}_{i}=$ $\left(\cos \left(\frac{\pi}{2} \varphi_{i}\right), \sin \left(\frac{\pi}{2} \varphi_{i}\right)\right)$, where $\left(\varphi_{1}, \ldots, \varphi_{6}\right)=(0,1,2,3,0.1,0.2)$ (see Fig. 11). Since all neighbors have the same distance from $\boldsymbol{x}_{0}$, the distance weight function does not play a role. Formula (8) yields the non-positive least squares stencil $s=(0.846,1.005,0.998,1.003,0.312,-0.164)$. However, the configuration admits a positive stencil, namely $s=(1,1,1,1,0,0)$.

\section{$5 \quad$ Linear Minimization Approach}

Least squares approaches do not guarantee positive stencils. As motivated in Sect. 2.2, we wish to allow positive stencils only. Hence, we enforce positivity, i.e. we search for solutions in the polyhedron

$$
P=\left\{s \in \mathbb{R}^{m}: V \cdot s=\boldsymbol{b}, \boldsymbol{s} \geq 0\right\} .
$$

This is the feasibility problem of linear optimization [19. In Sect. 6] we show under which conditions solutions exist. If $P$ is nonvoid and not degenerate, there are infinitely many feasible stencils. To single out a unique stencil we formulate a linear minimization problem

$$
\min \sum_{i=1}^{m} \frac{s_{i}}{w_{i}}, \text { s.t. } V \cdot s=b, s \geq 0,
$$

where the weights $w_{i}=w\left(\left\|\boldsymbol{x}_{i}-\boldsymbol{x}_{0}\right\|\right)$ are defined by an appropriately decaying (see Thm. 6) non-negative distance weight function $w$. Problem (10) is a linear program (LP) in standard form. It is bounded, since we have imposed sign constraints and the weights $w_{i}$ are all non-negative.

Theorem 5. If the polyhedron (9) is nonvoid, then the linear minimization approach (10) yields minimal positive stencils.

Proof. The sign constraints in (10) ensure that the selected stencil is positive. The existence of a minimal solution is ensured by the fundamental theorem of linear programming [19]. If the LP (10) has a solution, then it also has a basic solution, in which at most $k$ of the $m$ stencil entries $s_{i}$ are different from zero. 


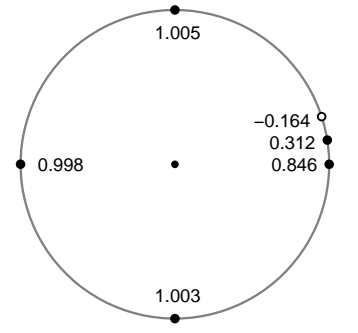

Figure 1: Non-positive LSQ stencil
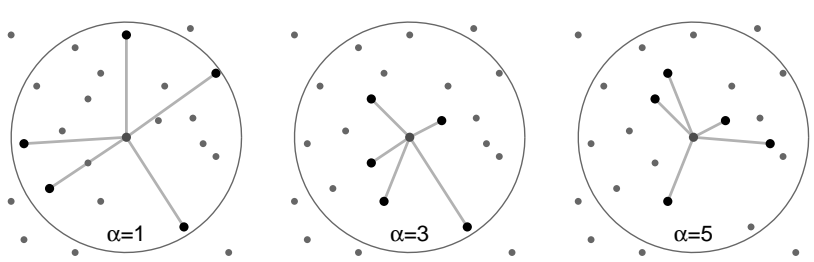

Figure 2: Minimal positive stencil for various values of $\alpha$

In contrast to least squares methods, the LP approach yields nonzero values only for a few selected points, and a continuous dependence on the point positions is not possible (see Rem. 1).

Remark 5. One could ask why not remain with a least squares problem, and additionally impose sign constraints. While this would be a valid approach (the solution is obtained by Karush-Kuhn-Tucker methods [19]), it would have the worst of both worlds. The solution would not depend continuously on the point cloud geometry whenever the sign constraints are active, and the resulting stencil would not be minimal. When sign constraints are imposed, linear minimization is preferable.

\subsection{Solving the Linear Programs}

For every interior point consider a set of candidate points $(m>k)$. A basic solution of (10) is computed. Only the nonzero stencil values enter the Poisson matrix. We refer to this approach as minimal positive stencil (MPS) method. The LPs (10) are small, but they have to be solved for every interior point. To our knowledge, there are no general results about efficient methods for such small LPs, especially considering the special structure of the Vandermonde matrix. A numerical comparison of various methods has been presented in [15, p. 148]. Simplex methods perform best for the arising LPs. A basis change corresponds to one stencil point replacing another. The theoretical worst case performance of simplex methods is not observed. Typical runs find the solution in about $1.5 k$ steps, resulting in a complexity of $O\left(k^{2} m\right)$, which equals the effort of least squares approaches (see Sect. 4). 


\subsection{Geometric Interpretation of Minimal Positive Stencils}

The MPS method forms a compromise between selecting neighbors close by and distributed nicely (see Def. 10) around the central point. How much preference is given to which objective depends on the locality parameter $\alpha$ in the distance weight function $w(\delta)=|\delta|^{-\alpha}$.

Theorem 6. The MPS method (10) only leads to reasonable results, if the distance weight function decays faster than $|\delta|^{-2}$.

Proof. Summing over the diagonal in the quadratic constraints in (3) yields the relation $\sum_{i=1}^{m}\left\|\overline{\boldsymbol{x}}_{i}\right\|_{2}^{2} s_{i}=2 d$. If $w(\delta)$ decays faster than $|\delta|^{-2}$, points close to the central point are given preference. If $w(\delta)=|\delta|^{-2}$, the LP (10) is degenerate. If $w(\delta)$ decays slower than $|\delta|^{-2}$, the approach selects points far away from the central point, possibly resulting in "checkerboard" instabilities.

The dependence of the MPS stencil on $\alpha$ is shown in Fig. $2^{2}$ From the candidate points in the circle, five neighbors are selected. While for $\alpha=1$ far away points are selected, $\alpha \in\{3,5\}$ yields nearby points. For $\alpha=3$ smaller angles are more important, for $\alpha=5$ smaller distances. Note that the MPS method never selects neighbors which are not distributed around the central point (as defined in Sect. 6), even if those are the $k$ closest points.

Remark 6. For regular grids, the MPS method selects standard finite difference stencils. For instance, for a regular Cartesian grid, the standard 5 -point $(2 \mathrm{~d})$, respectively 7 -point $(3 \mathrm{~d})$ stencils are obtained. In these cases, the basic solution is degenerate, i.e. some of the basis variables are zero.

\subsection{Neighborhood Criteria}

The circular neighborhood criterion yields a large number of neighbors (unless $r$ is very small). Also, it does not guarantee positive stencils, as the example in Fig. 3 shows. The selected neighbors are marked bold. Neighbors with non-positive stencil values are indicated by a white center. The presented methods can also be based on other neighborhood criteria.

Defining a neighborhood via the a Delaunay triangulation (tetrahedrization in 3d) [18, yields significantly fewer neighbors. However, the construction is a meshing procedure, hence it is often undesirable in a meshfree

\footnotetext{
${ }^{2}$ The figures are $2 \mathrm{~d}$ for simplicity of presentation. The MPS method applies directly to, and shows its strength, in $3 \mathrm{~d}$.
} 


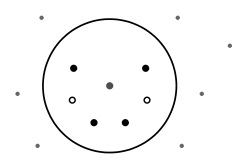

$\cdot$

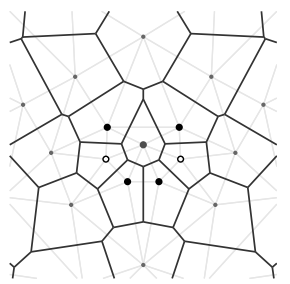

Figure 4: Delaunay neighbors

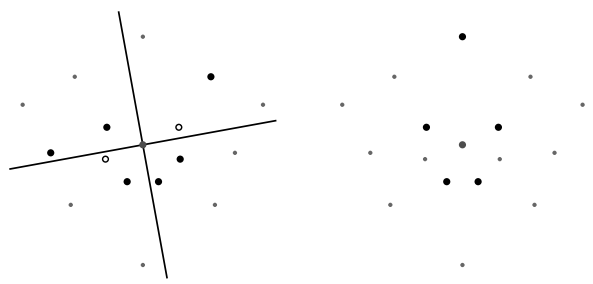

Figure 5: Four Figure 6: MPS quadrants neighborhood

lar neighborhood

context 3 Fig. 4 shows that a Delaunay neighborhood constructed from a Voronoi tessellation may yield non-positive stencils. In addition, it is possible that not enough neighbors are selected. If, in the example, the two negative points were removed, only four neighbors would be defined.

The four quadrant criterion [3] (eight sectors in 3d) defines a local coordinate system and selects the two closest points from each sector. It guarantees the neighbors to be distributed around the central point. However, it does not guarantee positive stencils, as the example in Fig. 5 shows.

The stencil selected by the MPS method is shown in Fig. 6. It is minimal and positive, here achieving this property by selecting one point further away. The MPS method can be interpreted as a neighborhood criterion that is optimal (i.e. minimal and positive) with respect to the Laplace operator. A deeper discussion of various neighborhood criteria in presented in [15].

\section{Conditions for the Existence of Positive Stencils}

We investigate when the polyhedron (91) is nonvoid, i.e. under which conditions positive stencils exist. We place the point of approximation in the origin $\boldsymbol{x}_{0}=\mathbf{0}$. A set of neighbors $\left\{\boldsymbol{x}_{1}, \ldots, \boldsymbol{x}_{m}\right\} \subset \mathbb{R}^{d}$ is given, where $m \geq k$. In order to establish a connection between the LP space $\mathbb{R}^{m}$ and the actual geometry space $\mathbb{R}^{d}$ we consider the dual problem, which is defined in $\mathbb{R}^{k}$.

Theorem 7 (Farkas' Lemma). For a real matrix $A$ and a real vector $\boldsymbol{b}$, exactly one of the following two systems has a solution:

- $A \cdot \boldsymbol{x}=\boldsymbol{b}$ for some $\boldsymbol{x} \geq 0$, or

- $A^{T} \cdot \boldsymbol{w} \geq 0$ for some $\boldsymbol{w}$ satisfying $\boldsymbol{b}^{T} \cdot \boldsymbol{w}<0$.

\footnotetext{
${ }^{3}$ Hybrid approaches exist that use a Delaunay mesh combined with meshfree methods.
} 
Proof. The proof is given in [19].

Applying Farkas' lemma to our problem yields that system $V \cdot \boldsymbol{s}=\boldsymbol{b}$ has no solution $s \geq 0$, if and only if system $V^{T} \cdot \boldsymbol{w} \geq 0$ has a solution satisfying $\boldsymbol{b}^{T} \cdot \boldsymbol{w}<0$. The $i^{\text {th }}$ component of $V^{T} \cdot \boldsymbol{w}$ can be written as

$$
\left(V^{T} \cdot \boldsymbol{w}\right)_{i}=\boldsymbol{a}^{T} \cdot \boldsymbol{x}_{i}+\boldsymbol{x}_{i}^{T} \cdot A \cdot \boldsymbol{x}_{i}
$$

where $\boldsymbol{a}=\left(w_{1}, \ldots, w_{d}\right)^{T}$ and $A$ is the symmetric matrix

$$
A=\left(\begin{array}{ll}
w_{4} & w_{3} \\
w_{3} & w_{5}
\end{array}\right)(2 \mathrm{~d}) \quad \text { resp. } \quad A=\left(\begin{array}{lll}
w_{7} & w_{4} & w_{5} \\
w_{4} & w_{8} & w_{6} \\
w_{5} & w_{6} & w_{9}
\end{array}\right) \quad(3 \mathrm{~d})
$$

Given $\boldsymbol{w}$ (respectively $\boldsymbol{a}$ and $A$ ), we consider the quadratic form $f(\boldsymbol{x})=$ $\boldsymbol{a}^{T} \cdot \boldsymbol{x}+\boldsymbol{x}^{T} \cdot A \cdot \boldsymbol{x}$. Since $A$ is symmetric, an orthogonal matrix $S \in O(d)$ exists, such that $S^{T} A S=D$, where $D=\operatorname{diag}\left(\lambda_{1}, \ldots, \lambda_{d}\right)$. In the new coordinates, with $\boldsymbol{d}=S^{T} \boldsymbol{a}$, we define

$$
g(\boldsymbol{x})=f(S \boldsymbol{x})=\boldsymbol{d}^{T} \cdot \boldsymbol{x}+\boldsymbol{x}^{T} \cdot D \cdot \boldsymbol{x} .
$$

If all eigenvalues $\lambda_{i} \neq 0$, then $D$ is regular. With $\boldsymbol{c}=-\frac{1}{2} D^{-1} \boldsymbol{d}$ we can write

$$
g(\boldsymbol{x})=(\boldsymbol{x}-\boldsymbol{c})^{T} \cdot D \cdot(\boldsymbol{x}-\boldsymbol{c})-\boldsymbol{c}^{T} \cdot D \cdot \boldsymbol{c},
$$

If one or two $\lambda_{i}=0$, we are in a degenerate case, and stick to the representation with $\boldsymbol{d}$ as parameter. Choosing $\boldsymbol{w} \in \mathbb{R}^{k}$ arbitrarily is equivalent to choosing $S \in O(d), \boldsymbol{\lambda}=\left(\lambda_{1}, \ldots, \lambda_{d}\right) \in \mathbb{R}^{d}$ and $\boldsymbol{c} \in \mathbb{R}^{d}$ (respectively $\boldsymbol{d} \in \mathbb{R}^{d}$ ) arbitrarily. For any $\boldsymbol{\lambda}, \boldsymbol{c} \in \mathbb{R}^{d}$ define the domain

$$
H_{\boldsymbol{\lambda}, \boldsymbol{c}}=\left\{\boldsymbol{x} \in \mathbb{R}^{d}: g(\boldsymbol{x}) \geq 0\right\} .
$$

For a set of points $X=\left\{\boldsymbol{x}_{1}, \ldots, \boldsymbol{x}_{m}\right\}$ define $S X=\left\{S \boldsymbol{x}_{1}, \ldots, S \boldsymbol{x}_{m}\right\}$. Farkas' lemma translates to

Corollary 1. System $V \cdot \boldsymbol{s}=\boldsymbol{b}$ has no solution $\boldsymbol{s} \geq 0$, if and only if $S \in O(d), \boldsymbol{c}, \boldsymbol{\lambda} \in \mathbb{R}^{d}$ with $\sum_{i=1}^{d} \lambda_{i}<0$ exist, such that $S X \subset H_{\boldsymbol{\lambda}, \boldsymbol{c}}$.

In other words, no positive Laplace stencil exists, iff the set of points $X$ can be transformed (via $S \in O(d)$ ), such that it is contained in the set $H_{\boldsymbol{\lambda}, \boldsymbol{c}}$ for some $\boldsymbol{c}, \boldsymbol{\lambda} \in \mathbb{R}^{d}$ with $\sum_{i=1}^{d} \lambda_{i}<0$.

Example 2. The setup in Fig. 7 shows a set of points that is completely contained in a domain $H_{\boldsymbol{\lambda}, \boldsymbol{c}}$. Due to Cor. 1, no positive stencil exists. 


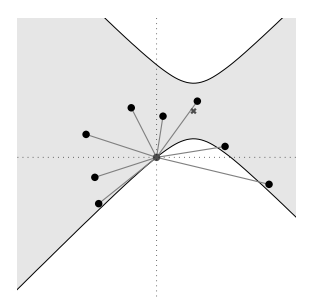

Figure 7: No positive stencil exists

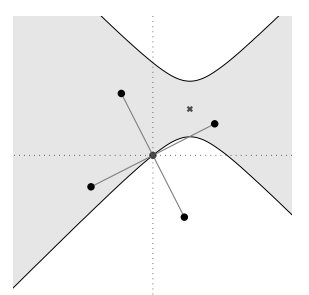

Figure 8: Positive stencil

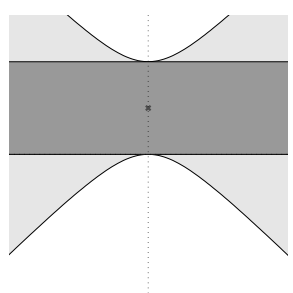

Figure 9: Necessary criterion

Example 3. The setup in Fig. 8 shows a point setup which has a positive stencil solution. It is impossible to find a domain $H_{\boldsymbol{\lambda}, \boldsymbol{c}}$ and to rotate the set of points, such that all points are contained in the domain.

We have derived a geometric condition, which is equivalent to the existence of positive stencils. However, due to the nonlinearity in $g$, it is difficult to translate into geometric means directly. Instead, we derive a necessary (but not sufficient) as well as a sufficient (but not necessary) criterion on the point geometry for the existence of a positive Laplace stencil. To our knowledge the latter has not been given yet.

\subsection{A Necessary Criterion for Positive Stencils}

If $V \cdot \boldsymbol{s}=\boldsymbol{b}$ has a solution $\boldsymbol{s} \geq 0$, then for any $S \in O(d), \boldsymbol{c}, \boldsymbol{\lambda} \in \mathbb{R}^{d}$ with $\sum_{i=1}^{d} \lambda_{i}<0$, there is a point $\boldsymbol{x}_{i}$ with $S \boldsymbol{x}_{i} \notin H_{\boldsymbol{\lambda}, \boldsymbol{c}}$. For the particular choice $\lambda_{1}=-1, \lambda_{i}=0 \forall i>1$ and $c_{1} \gg \max _{i}\left\|\boldsymbol{x}_{i}\right\|$ it follows that for any $S \in O(d)$ at least one point must satisfy $x_{1}<0$. This yields the following

Theorem 8. If a set of points $X \subset \mathbb{R}^{d}$ around the origin admits a positive Laplace stencil, then they must not lie in one and the same half space (with respect to an arbitrary hyperplane through the origin).

This result is well known [3]. Due to the particular choice of $\boldsymbol{\lambda}$, this criterion is very crude, but easy to formulate in geometric means. More careful estimates of the condition of Cor. 1 may yield stricter criteria.

\subsection{A Sufficient Criterion for Positive Stencils}

For any $\boldsymbol{c}, \boldsymbol{\lambda} \in \mathbb{R}^{d}$ with $\sum_{i=1}^{d} \lambda_{i}<0$ we construct a domain $G_{\boldsymbol{\lambda}, \boldsymbol{c}} \supset H_{\boldsymbol{\lambda}, \boldsymbol{c}}$, which is $\mathbb{R}^{d}$ aside from a cone centered at the origin. If for any $\boldsymbol{c}, \boldsymbol{\lambda} \in \mathbb{R}^{d}$, $S \in O(d)$ there is at least one point $S \boldsymbol{x}_{i} \notin G_{\boldsymbol{\lambda}, \boldsymbol{c}}$, then $S \boldsymbol{x}_{i} \notin H_{\boldsymbol{\lambda}, \boldsymbol{c}}$, thus a positive Laplace stencil exists. We call this criterion cone criterion. 

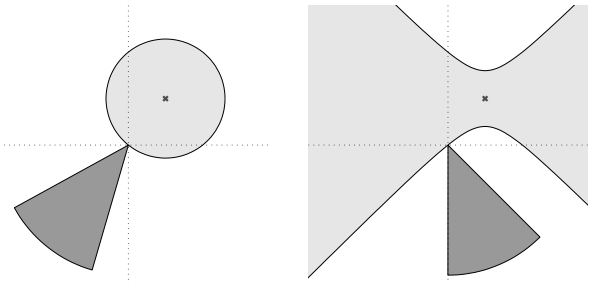

Figure 10: Case Figure 11: Case $(--)$

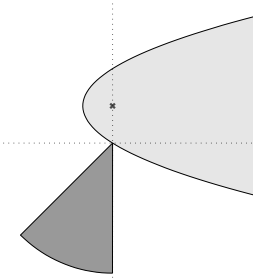

Figure 12: Case (0-) type 1

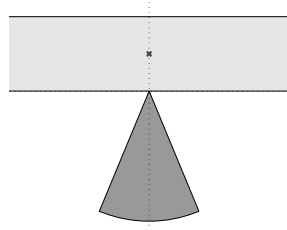

Figure 13: Case (0-) type 2

Theorem 9 (Cone criterion in $2 \mathrm{~d}$ ). Let $\boldsymbol{c}, \boldsymbol{\lambda} \in \mathbb{R}^{2}$ with $\lambda_{1}+\lambda_{2}<0$. There exists always a cone $C \boldsymbol{v}$ defined by $\boldsymbol{v} \cdot \boldsymbol{x}>\frac{1}{\sqrt{1+\beta^{2}}}\|\boldsymbol{x}\|$, where $\beta=\sqrt{2}-1$ (a cone with total opening angle $45^{\circ}$, the direction vector $\boldsymbol{v}$ depends on $\boldsymbol{\lambda}$ and c), such that $G_{\boldsymbol{\lambda}, \boldsymbol{c}}=\mathbb{R}^{d} \backslash C_{\boldsymbol{v}}$ satisfies $H_{\boldsymbol{\lambda}, \boldsymbol{c}} \subset G_{\boldsymbol{\lambda}, \boldsymbol{c}}$.

Proof. We show that $H_{\boldsymbol{\lambda}, \boldsymbol{c}}$ and $C_{\boldsymbol{v}}$ do not intersect. Since the problem is invariant under interchanging coordinates, we can w.l.o.g. assume that $\lambda_{2}<0$. Including the degenerate case, three cases need to be considered:

- Case (--): $\lambda_{1}<0, \lambda_{2}<0$

The set $H_{\boldsymbol{\lambda}, \boldsymbol{c}}$ is the interior of an ellipse centered at $\boldsymbol{c}$ with $\mathbf{0} \in \partial H_{\boldsymbol{\lambda}, \boldsymbol{c}}$. The vector $\boldsymbol{v}=-\left(\frac{\lambda_{2}}{\lambda_{1}} c_{1}, \frac{\lambda_{1}}{\lambda_{2}} c_{2}\right)$ is an outer normal vector. The cone $C \boldsymbol{v}$ touches the ellipse only at the origin, as shown in Fig. 10.

- Case $(+-): \lambda_{1}>0, \lambda_{2}<0$

Fig. 11 shows the geometry. Define $\mu_{1}=\frac{\left|\lambda_{1}\right|}{\lambda_{2} \mid}<1$. The domain $H_{\boldsymbol{\lambda}, \boldsymbol{c}}$ is defined by $\tilde{g}\left(x_{1}, x_{2}\right)=\mu_{1}\left(x_{1}^{2}-2 c_{1} x_{1}\right)-\left(x_{2}^{2}-2 c_{2} x_{2}\right) \geq 0$. Due to symmetry we can assume $c_{1}, c_{2} \geq 0$. For all $\boldsymbol{x} \in B$, where $B=\left\{\left(x_{1}, x_{2}\right)\left|x_{1}>0, x_{2}<0,\right| x_{1}|<| x_{2} \mid\right\}$, the function $\tilde{g}$ satisfies

$$
\begin{aligned}
\tilde{g}(\boldsymbol{x}) & =\mu_{1}\left(\left|x_{1}\right|^{2}-2 c_{1}\left|x_{1}\right|\right)-\left(\left|x_{2}\right|^{2}+2 c_{2}\left|x_{2}\right|\right) \\
& <\left(\mu_{1}-1\right)\left|x_{2}\right|^{2}-2\left(\mu_{1} c_{1}\left|x_{1}\right|+c_{2}\left|x_{2}\right|\right)<0,
\end{aligned}
$$

hence $H_{\boldsymbol{\lambda}, \boldsymbol{c}} \cap B=\emptyset$. The domain $B$ is a $2 \mathrm{~d}$ cone with opening angle $45^{\circ}$, where $\boldsymbol{v}=\left(\frac{1}{2} \sqrt{2-\sqrt{2}}, \frac{1}{2} \sqrt{2+\sqrt{2}}\right)$, which proves the claim.

- Case (0-): $\lambda_{1}=0, \lambda_{2}<0$

We use representation (11). Define $\mu_{2}=\left|\lambda_{2}\right|$. The domain $H_{\boldsymbol{\lambda}, \boldsymbol{d}}$ is defined by $g\left(x_{1}, x_{2}\right)=d_{1} x_{1}+d_{2} x_{2}-\mu_{2} x_{2}^{2} \geq 0$. Due to symmetry we can assume that $d_{1}, d_{2} \geq 0$. Two subcases have to be distinguished: 
- Case $d_{1} \neq 0$ :

The setup is shown in Fig. 12, For all $\boldsymbol{x} \in B$, where $B=$ $\left\{\left(x_{1}, x_{2}\right)\left|x_{1}<0, x_{2}<0,\right| x_{1}|<| x_{2} \mid\right\}$, one has $g\left(x_{1}, x_{2}\right)=-d_{1}\left|x_{1}\right|-$ $d_{2}\left|x_{2}\right|-\mu_{2} x_{2}^{2}<0$, hence $H_{\boldsymbol{\lambda}, \boldsymbol{d}} \cap B=\emptyset$. As above, the domain $B$ is a $2 \mathrm{~d}$ cone with opening angle $45^{\circ}$.

- Case $d_{1}=0$ :

The setup is shown in Fig. 13. The domain $g\left(x_{1}, x_{2}\right) \geq 0$ is the set $0 \leq x_{2} \leq \frac{d_{2}}{\mu_{2}}$. Any cone contained in the domain $x_{2}<0$ proves the claim.

In other words, if any angle between two neighboring points (seen from the central point) is no more than $45^{\circ}$, then a positive stencil always exists.

Remark 7 . The $2 \mathrm{~d}$ cone criterion is sharp: For any $\varepsilon>0$ a point setup can be constructed, such that all angles are less than $\frac{\pi}{4}+\varepsilon$, and a positive stencil does not exist. The construction is given in [15, p. 136]. Note that the resulting configurations are very unbalanced. Some points are very close to the central point, others are far away. In practice, such extreme cases are typically avoided by the construction and management of the point cloud, yielding positive stencils also for angles significantly larger than $45^{\circ}$.

Theorem 10 (Cone criterion in 3d). Let $\boldsymbol{c}, \boldsymbol{\lambda} \in \mathbb{R}^{3}$ with $\lambda_{1}+\lambda_{2}+\lambda_{3}<$ 0 . There exists always a cone $C \boldsymbol{v}$ defined by $\boldsymbol{v} \cdot \boldsymbol{x}>\frac{1}{\sqrt{1+\beta^{2}}}\|\boldsymbol{x}\|$, where $\beta=\sqrt{\frac{1}{6}(3-\sqrt{6})}$ (a cone with total opening angle 33.ๆ ), such that $G_{\boldsymbol{\lambda}, \boldsymbol{c}}=$ $\mathbb{R}^{d} \backslash C_{\boldsymbol{v}}$ satisfies $H_{\boldsymbol{\lambda}, \boldsymbol{c}} \subset G_{\boldsymbol{\lambda}, \boldsymbol{c}}$.

Proof. The following cases need to be considered:

- Cases $(---),(0--),(00-): \lambda_{1} \leq 0, \lambda_{2} \leq 0, \lambda_{3}<0$

As in $2 \mathrm{~d}$, we use representation (11). Define $\mu_{i}=\left|\lambda_{i}\right| \forall i=1,2,3$. Allowing $\mu_{1}$ and $\mu_{2}$ to be zero, the domain $H_{\boldsymbol{\lambda}, \boldsymbol{d}}$ is defined by

$$
g\left(x_{1}, x_{2}, x_{3}\right)=d_{1} x_{1}+d_{2} x_{2}+d_{3} x_{3}-\mu_{1} x_{1}^{2}-\mu_{2} x_{2}^{2}-\mu_{3} x_{3}^{2} \geq 0,
$$

First assume that if one $\mu_{i}=0$, then the corresponding $d_{i} \neq 0$. Due to symmetry we can w.l.o.g. assume that $d_{1}, d_{2}, d_{3} \geq 0$. For all $\boldsymbol{x} \in B$, where $B=\left\{\left(x_{1}, x_{2}, x_{3}\right) \mid x_{1}, x_{2}, x_{3}<0\right\}$, the function $g$ satisfies

$$
g\left(x_{1}, x_{2}, x_{3}\right)=-d_{1}\left|x_{1}\right|-d_{2}\left|x_{2}\right|-d_{3}\left|x_{3}\right|-\mu_{2}\left|x_{2}\right|^{2}-\mu_{3}\left|x_{3}\right|^{2}<0 .
$$

The domain $B$ is not a cone, but the corresponding domain from the case $(++-)$ is contained in it. Hence, the cone constructed in that case can be used here. 
In the case that $\mu_{i}=0$ and $d_{i}=0$, the geometry reduces to the $2 \mathrm{~d}$ (or trivial 1d) case. Since in $2 \mathrm{~d}$ the desired estimates have been shown for a larger opening angle, the constructions transfer to the $3 \mathrm{~d}$ case.

- Case $(++-): \lambda_{1}>0, \lambda_{2}>0, \lambda_{3}<0$

Define $\mu_{1}=\frac{\left|\lambda_{1}\right|}{\left|\lambda_{3}\right|}<1$ and $\mu_{2}=\frac{\left|\lambda_{2}\right|}{\left|\lambda_{3}\right|}<1$. Then $H_{\boldsymbol{\lambda}, \boldsymbol{c}}$ is defined by

$$
\tilde{g}\left(x_{1}, x_{2}, x_{3}\right)=\mu_{1}\left(x_{1}^{2}-2 c_{1} x_{1}\right)+\mu_{2}\left(x_{2}^{2}-2 c_{2} x_{2}\right)-\left(x_{3}^{2}-2 c_{3} x_{3}\right) \geq 0 .
$$

Due to symmetry we can assume $c_{1}, c_{2}, c_{3} \geq 0$. For all $\boldsymbol{x} \in B$, where $B=\left\{\left(x_{1}, x_{2}, x_{3}\right)\left|x_{1}, x_{2}>0, x_{3}<0,\right| x_{1}|,| x_{2}\left|<\sqrt{\frac{1}{2}}\right| x_{3} \mid\right\}$, we have

$$
\begin{aligned}
\tilde{g}(\boldsymbol{x}) & =\mu_{1}\left(\left|x_{1}\right|^{2}-2 c_{1}\left|x_{1}\right|\right)+\mu_{2}\left(\left|x_{2}\right|^{2}-2 c_{2}\left|x_{2}\right|\right)-\left(\left|x_{3}\right|^{2}+2 c_{3}\left|x_{3}\right|\right) \\
& <\left(\frac{1}{2}\left(\mu_{1}+\mu_{2}\right)-1\right)\left|x_{3}\right|^{2}-2\left(\mu_{1} c_{1}\left|x_{1}\right|+\mu_{2} c_{2}\left|x_{2}\right|+c_{3}\left|x_{3}\right|\right)<0 .
\end{aligned}
$$

Note that $B$ is not a cone. However, a $3 \mathrm{~d}$ cone can always be contained inside $B$. Some geometric considerations yield that the cone with maximum opening angle contained inside $B$ is given by $\beta=\sqrt{\frac{1}{6}(3-\sqrt{6})}$ and $\boldsymbol{v}=\frac{1}{\sqrt{41-16 \sqrt{6}}}(2(\sqrt{3}-\sqrt{2}), 2(\sqrt{3}-\sqrt{2}), 1)$.

- Case $(+0-): \lambda_{1}>0, \lambda_{2}=0, \lambda_{3}<0$

As in the preceding degenerate cases, we describe the domain by (11). Define $\mu_{1}=\left|\lambda_{1}\right|$ and $\mu_{3}=\left|\lambda_{3}\right|$. The case $d_{2}=0$ reduces to the $2 \mathrm{~d}$ case $(+-)$. Hence, w.l.o.g. we consider $d_{1} \geq 0, d_{2}>0, d_{3} \geq 0$. For all $\boldsymbol{x} \in B$, where $B=\left\{\left(x_{1}, x_{2}, x_{3}\right)\left|x_{1}, x_{2}, x_{3}<0,\right| x_{1}|<| x_{3} \mid\right\}$, we have

$$
g\left(x_{1}, x_{2}, x_{3}\right)=-d_{1}\left|x_{1}\right|-d_{2}\left|x_{2}\right|-d_{3}\left|x_{3}\right|+\mu_{1}\left|x_{1}\right|^{2}-\mu_{3}\left|x_{3}\right|^{2}<0 .
$$

The estimate holds, since $\mu_{1}<\mu_{3}$ and $\left|x_{1}\right|<\left|x_{3}\right|$. As before, a 3d cone with desired opening angle can be contained in $B$. The cone from the case $(++-)$ can be used here.

- Case $(+--): \lambda_{1}>0, \lambda_{2}<0, \lambda_{3}<0$

Define $\mu_{2}=\frac{\left|\lambda_{2}\right|}{\left|\lambda_{1}\right|}$ and $\mu_{3}=\frac{\left|\lambda_{3}\right|}{\left|\lambda_{1}\right|}$. Since $\mu_{2}+\mu_{3}>1$, we assume w.l.o.g. $\mu_{3} \geq \frac{1}{2}$. The domain $H_{\boldsymbol{\lambda}, \boldsymbol{c}}$ is defined by

$$
\tilde{g}\left(x_{1}, x_{2}, x_{3}\right)=\left(x_{1}^{2}-2 c_{1} x_{1}\right)-\mu_{2}\left(x_{2}^{2}-2 c_{2} x_{2}\right)-\mu_{3}\left(x_{3}^{2}-2 c_{3} x_{3}\right) \geq 0 .
$$


Due to symmetry we can assume $c_{1}, c_{2}, c_{3} \geq 0$. For all $\boldsymbol{x} \in B$, where $B=\left\{\left(x_{1}, x_{2}, x_{3}\right)\left|x_{1}>0, x_{2}, x_{3}<0,\right| x_{1}|,| x_{2}\left|<\sqrt{\frac{1}{2}}\right| x_{3} \mid\right\}$ we have

$$
\begin{aligned}
\tilde{g}(\boldsymbol{x}) & =\left(\left|x_{1}\right|^{2}-2 c_{1}\left|x_{1}\right|\right)-\mu_{2}\left(\left|x_{2}\right|^{2}+2 c_{2}\left|x_{2}\right|\right)-\mu_{3}\left(\left|x_{3}\right|^{2}+2 c_{3}\left|x_{3}\right|\right) \\
& =\underbrace{\left(\left|x_{1}\right|^{2}-\mu_{2}\left|x_{2}\right|^{2}-\mu_{3}\left|x_{3}\right|^{2}\right)}_{<\left(\frac{1}{2}-\mu_{3}\right)\left|x_{3}\right|^{2} \leq 0}-2\left(\mu_{1} c_{1}\left|x_{1}\right|+\mu_{2} c_{2}\left|x_{2}\right|+c_{3}\left|x_{3}\right|\right)<0
\end{aligned}
$$

The domain $B$ is the same as in case $(++-)$, merely reflected at the $x_{1}, x_{3}$ plane. Hence, a $3 \mathrm{~d}$ cone can be placed in the same way.

Remark 8. Unlike the $2 \mathrm{~d}$ case, the $3 \mathrm{~d}$ estimate is not sharp, due to the intermediate domain $B$. With significantly more algebra, it is possible to gain an opening angle that is a couple of degrees larger.

Remark 9. The existence of a positive stencil implies the existence of a stencil. Configurations that yield an unsolvable Vandermonde system (4) are automatically excluded by the cone criterion.

Definition 10. We call points distributed nicely around a central point, if in a test cone, with opening angle given by Thm. 9 respectively Thm. 10, always points are contained, for any possible direction the cone points to.

\subsection{Condition on Point Cloud Geometry}

The cone criterion guarantees positive stencils. We now provide conditions on the point cloud geometry and the choice of candidate points, such that the cone criterion is guaranteed to be satisfied. As in [10], we define

Definition 11. Let $\Omega \subset \mathbb{R}^{d}$ be a domain and $X=\left\{\boldsymbol{x}_{1}, \ldots, \boldsymbol{x}_{n}\right\}$ a point cloud. The mesh size $h$ is defined as the minimal real number, such that $\bar{\Omega} \subset \bigcup_{i=1}^{n} \bar{B}\left(\boldsymbol{x}_{i}, \frac{h}{2}\right)$, where $\bar{B}(\boldsymbol{x}, r)$ is the closed ball of radius $r$ centered in $\boldsymbol{x}$ and $\bar{\Omega}$ is the closure of $\Omega$.

We assume that a desired maximum mesh size is preserved by management of the point cloud, e.g. by inserting points into large holes.

Theorem 11. Let the point cloud have mesh size $h$. Let $\gamma$ be the opening angle of the cone derived in Thm. 9 respectively Thm. 10. If the radius of considered candidate points satisfies $r>\frac{1}{\sin (\gamma / 2)} \frac{h}{2}$, then for every interior point which is sufficiently far from the boundary, a positive stencil exists. 


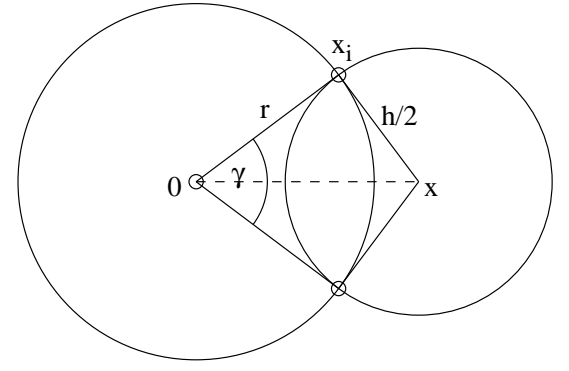

Figure 14: Relation between neighborhood radius and mesh size

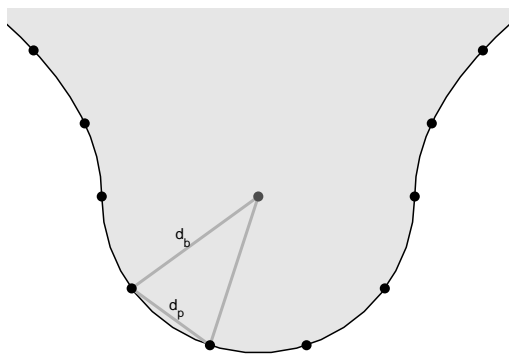

Figure 15: Guaranteeing the cone criterion close to the boundary

Proof. Having mesh size $h$ implies that there are no holes larger in diameter than $h$, i.e. $\forall \boldsymbol{x} \in \Omega \exists \boldsymbol{x}_{i} \in X:\left\|\boldsymbol{x}_{i}-\boldsymbol{x}\right\|<\frac{h}{2}$. Fig. 14 shows a ball with radius $r$ around the central point and a cone with opening angle $\gamma$. If the cone contains no point, there must be a ball of radius $\frac{h}{2}$ which contains no points. The claim follows by considering the triangle $\left(0, \boldsymbol{x}, \boldsymbol{x}_{i}\right)$.

The specific ratios of candidate radius to maximum hole size radius are

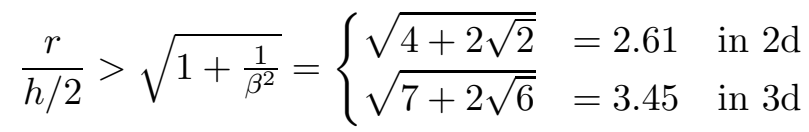

Using sharper estimates, the 3 d ratio can be lowered to $\sqrt{6+2 \sqrt{6}}=3.30$. In practice, point clouds are much nicer than the worst case scenario, so significantly smaller ratios lead to positive stencils.

Thm. 11 is valid for any interior point which is far enough from the boundary, that the mesh size criterion guarantees points to lie between the point in consideration and the boundary. For a layer of interior points close to the boundary, the cone criterion can be enforced by the following construction (see Fig. 15): First, place boundary points sufficiently dense. Let their maximum distance be $d_{\mathrm{p}}$. Second, ensure that every interior point has a minimum distance $d_{\mathrm{b}}$ from the boundary. In $2 \mathrm{~d}$, this is $d_{\mathrm{b}}>\frac{4}{\pi} d_{\mathrm{p}}$.

\subsection{Neumann Boundary Points}

Assume the boundary $\partial \Omega$ is $C^{1}$ around Neumann boundary points. Consider a local coordinate system, i.e. $\boldsymbol{n}=(1,0)$ in $2 \mathrm{~d}$, respectively $\boldsymbol{n}=(1,0,0)$ in 
3d. We obtain Neumann stencils by solving the linear minimization problem

$$
\min \sum_{i=1}^{m} \frac{s_{i}}{w_{i}}, \text { s.t. } V \cdot s=n, s \geq 0,
$$

where the matrix $V$ is given by (6) as

$$
V=\left(\begin{array}{ccc}
x_{1} & \ldots & x_{m} \\
y_{1} & \ldots & y_{m}
\end{array}\right) \text { in } 2 \mathrm{~d}, \text { and } V=\left(\begin{array}{ccc}
x_{1} & \ldots & x_{m} \\
y_{1} & \ldots & y_{m} \\
z_{1} & \ldots & z_{m}
\end{array}\right) \text { in } 3 \mathrm{~d} \text {. }
$$

We consider the $3 \mathrm{~d}$ case. The $2 \mathrm{~d}$ geometry is contained as a special case. For an easier analysis, we consider a locally convex domain, i.e. $x_{i} \geq 0 \forall i$.

Theorem 12. For a Neumann boundary point a positive stencil exists, iff the points' projections onto the normal plane do not lie all in one and the same half space.

Proof. Due to Farkas' lemma, system (12) has no solution $s \geq 0$, iff the system $V^{T} \cdot \boldsymbol{w} \geq 0$ has a solution satisfying $w_{x}<0$, where $\boldsymbol{w}=\left(w_{x}, w_{y}, w_{z}\right)^{T}$.

Let no positive stencil exist. Then $\boldsymbol{w} \in \mathbb{R}^{d}$ with $w_{x}<0$ exists, such that $V^{T} \cdot \boldsymbol{w} \geq 0$, i.e. $w_{x} x_{i}+w_{y} y_{i}+w_{z} z_{i} \geq 0 \forall i$. This is equivalent to $\boldsymbol{k} \cdot\left(\begin{array}{c}y_{i} \\ z_{i}\end{array}\right) \geq x_{i} \forall i$, where $\boldsymbol{k}=\left(\frac{w_{y}}{\left|w_{x}\right|}, \frac{w_{z}}{\left|w_{x}\right|}\right)$. This means that the $y$ - $z$ projection of all points lies in one and the same half space (in the direction of $\boldsymbol{k}$ ).

Conversely, assume that the $y-z$ projection of all points lies in one and the same half space. Let $I$ be the indices of all points in consideration. Define $I_{p}=\left\{i \in I: x_{i}>0\right\}$. Consider w.l.o.g. the case $z_{i} \geq 0 \forall i$, where $z_{i}>0 \forall i \in I_{p}$. Choose $\boldsymbol{w}=\left(-1,0, \frac{\max _{i \in I} x_{i}}{\min _{i \in I_{p}} z_{i}}\right)$. Then for all $i \in I_{p}$ it holds

$$
\boldsymbol{w}^{T} \cdot \boldsymbol{x}_{i}=-x_{i}+\frac{\max _{i \in I} x_{i}}{\min _{i \in I_{p}} z_{i}} z_{i} \geq-x_{i}+\max _{i \in I} x_{i} \geq 0,
$$

and for all $i \in I \backslash I_{p}$ one has $\boldsymbol{w}^{T} \cdot \boldsymbol{x}_{i} \geq 0$, since $x_{i}=0$. Thus, no positive stencil exists.

Remark 10. Construction (12) yields a first order accurate approximation of the normal derivative. Second order accuracy could be achieved, by including quadratic terms into $V$. However, in this case no positive stencil exists, since the condition $\sum_{i=1}^{m}\left(x_{i}^{2}+y_{i}^{2}\right) s_{i}=0$ cannot be satisfied. 


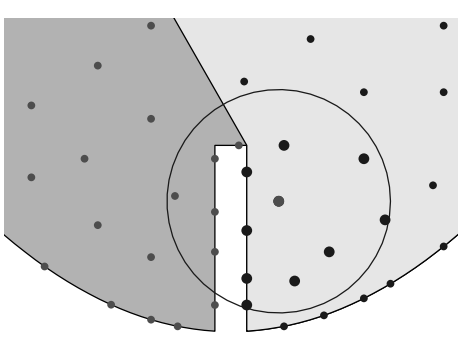

Figure 16: Proper treatment of a crack
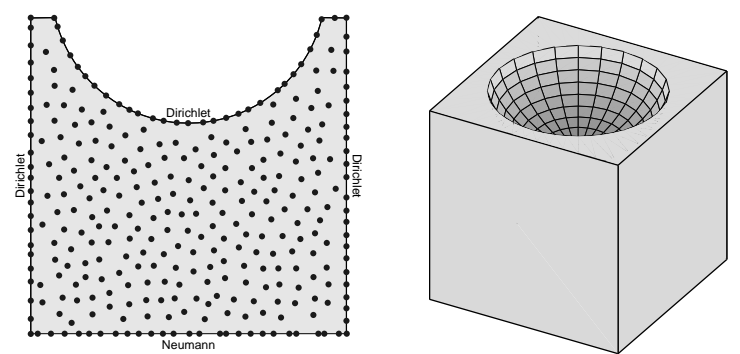

Figure 17: Computational domain in $2 \mathrm{~d}$ and $3 \mathrm{~d}$

\subsection{Treatment of Cracks}

A non-convex part of the domain $\Omega$ requires a special treatment if it is thinner than the local neighborhood radius. Fig. 16 shows a proper treatment of a crack. Stencils on one side of the crack must not use points on the other side. In the MPS method this property can be guaranteed by the following construction: For a central point $\boldsymbol{x}_{0} \in \Omega$, only circular neighbors inside the star shaped core $\bar{\Omega}_{\boldsymbol{x}_{0}}=\left\{\boldsymbol{x} \in \bar{\Omega}:(1-\alpha) \boldsymbol{x}_{0}+\alpha \boldsymbol{x} \in \bar{\Omega} \forall \alpha \in[0,1]\right\}$ (bold dots in Fig. 16) are considered as candidates for the linear minimization (10). If the domain is defined implicitly $\Omega=\{\boldsymbol{x}: \phi(\boldsymbol{x})<0\}$, the point $\boldsymbol{x}$ does not lie in $\bar{\Omega} \boldsymbol{x}_{0}$ if a point $\boldsymbol{y} \in\left[\boldsymbol{x}_{0}, \boldsymbol{x}\right]$ on the connection line satisfies $\phi(\boldsymbol{y})>0$.

\section{Minimal Stencils and Matrix Connectivity}

Due to Thm. 3 and Thm. 4, the matrix composed of positive stencils is an M-matrix, if every interior and Neumann boundary point is connected to a Dirichlet boundary point.

Theorem 13. Consider the Poisson problem (1) on a domain which has no holes (i.e. only an outer boundary). With a MPS discretization every interior point is connected to a boundary point.

Proof. Assume there is a point $i \in I$ which is not connected to a boundary point. Define $I_{i}=\{j \in I: i$ is connected to $j\}$. Every point in $I_{i}$ is not connected to a boundary point. Hence, the set $I_{i} \subset I$ forms an island inside $\Omega$ which does not reach a the boundary. Consider a point that spans the convex hull of $I_{i}$. It only uses points in its stencil that lie inside the island, hence these lie in one and the same half space, which contradicts the necessary condition on positive stencils given by Thm. 8 , 

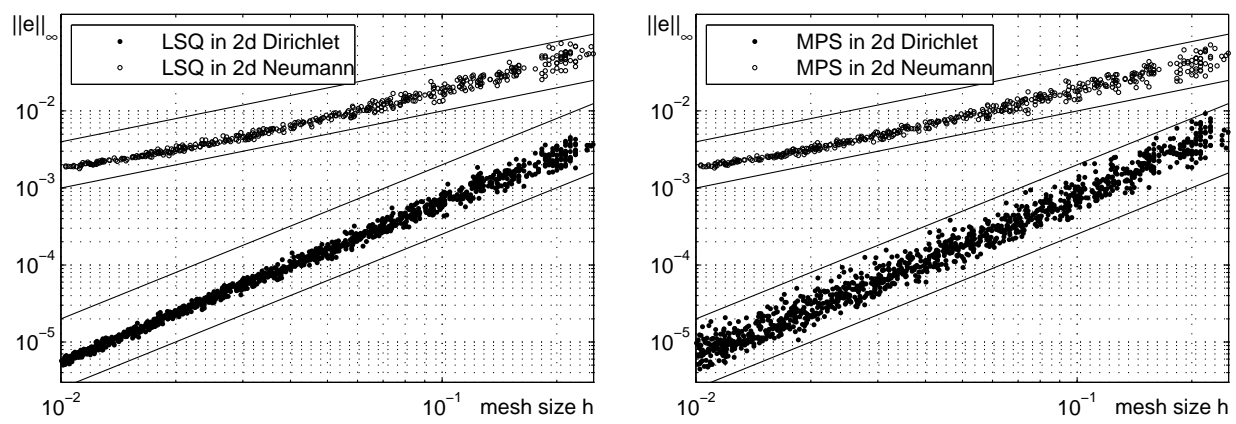

Figure 18: Error convergence in 2d - LSQ vs. MPS method

Remark 11. Although Thm. 13 does not extend to interior boundaries, in practice the MPS works for these, given enough boundary points are placed.

It remains to ensure that every point connects to a Dirichlet boundary point. Unfortunately, this cannot be concluded from the MPS method directly. It is possible that an isolated Dirichlet point is not used in the stencils of nearby interior points. Note that this phenomenon can also happen on regular grids. A single Dirichlet point in a corner of a domain may not be used by regular five-point stencils. If Dirichlet data is prescribed only in small regions, these regions have to be equipped with a sufficient number of boundary points. In addition, the MPS implementation has to ensure that these Dirichlet points are used by nearby points. If done so, the MPS method guarantees to generate M-matrices.

\section{Numerical Experiments}

We investigate the numerical accuracy of the MPS method in comparison to a least squares approach. As test problems we consider the Poisson equation (11) in the unit box with a ball cut out $\Omega=[0,1]^{d} \backslash B\left(\left(\frac{1}{2}, \ldots, \frac{1}{2}, 1.1\right), 0.44\right)$. Fig. 17 shows the computational domain in $2 \mathrm{~d}$ and $3 \mathrm{~d}$. In one case, the boundary conditions are Dirichlet everywhere, in the other case, Neumann at the bottom $x_{d}=0$, and Dirichlet everywhere else. Given $g$, we set $f=\Delta g$ and $h=\frac{\partial g}{\partial n}$, so (1) has the solution $u=g$. Specifically, we choose $g\left(x_{1}, x_{2}\right)=\frac{1}{c_{2}}\left(x_{1} \sin \left(x_{2}+2\right)+x_{2} \sin \left(2 x_{1}+1\right)\right)$ in $2 \mathrm{~d}$ and $g\left(x_{1}, x_{2}, x_{3}\right)=$ $\frac{1}{c_{3}}\left(x_{1} \sin \left(x_{2}+2\right)+x_{2} \sin \left(2 x_{3}+3\right)+x_{3} \sin \left(3 x_{1}+1\right)\right)$ in $3 \mathrm{~d}$, with $c_{2}$ and $c_{3}$ such that $\max g-\min g=1$. The problem is discretized by a sequence of point clouds. The point clouds have a uniform average density and a 

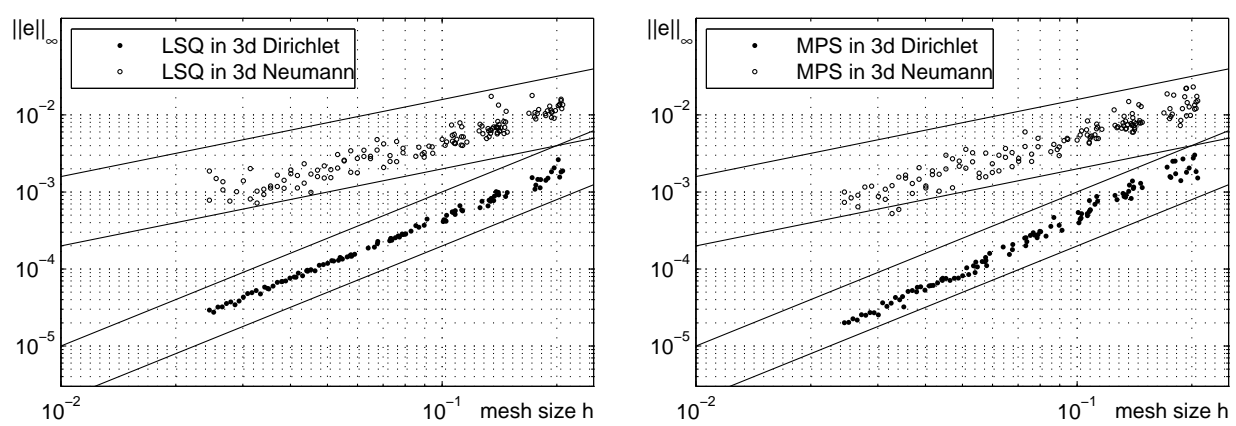

Figure 19: Error convergence in 3d - LSQ vs. MPS method

minimum separation [10] of $\delta=0.05$. Each point cloud is managed to satisfy the conditions for the existence of positive stencils, as derived in Sect. 6.3, Since to one mesh size $h$, given by Def. 11, many point clouds exist, we sample a number of experiments to obtain an average error convergence rate. To every point cloud we apply a weighted least squares method (Sect. 4) and the MPS methods (Sect. 5), both with $w(\delta)=\delta^{-4}$.

The numerical results are shown in Fig. 18 for 2d, and Fig. 19 for 3d. Plotted is the error measured in the maximum norm over the mesh size $h$. Solid dots represent the all Dirichlet version of a problem, while open circles show the error with partial Neumann boundary conditions. The reference lines are of slope one and two respectively. One can observe the following:

- Both approaches show a second order convergence rate for the pure Dirichlet problem, and first order convergence if Neumann boundary conditions are involved. While the derivation in Sect. 2.1 enforces only a first order accurate approximation of the Laplacian at interior points, point clouds tend to possess enough averaged symmetry to actually yield second order error convergence. On the other hand, the first order accurate approximation (Rem. 10) at Neumann boundary points carries through.

- The MPS method shows a larger variation in error over the ensemble of experiments. One reason for this effect could be the discontinuous dependence on the point positions (see Rem. 1). For two similar point clouds, the MPS method may select very different stencils.

- Both methods yield roughly the same error constant. The average error is slightly lower with the MPS method. 

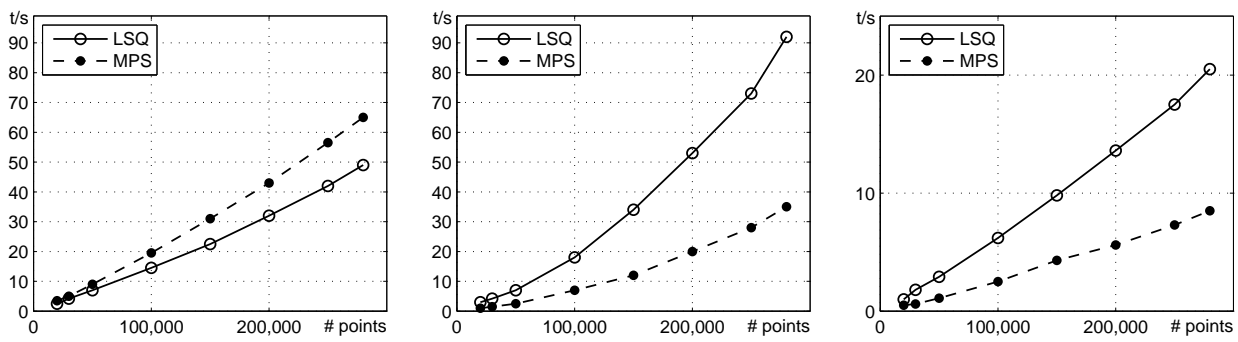

Figure 20: CPU times for setup and solve by BiCG and AMG

\subsection{Computational Cost}

For a 3d test problem, the MPS method is compared with the LSQ method in terms of computational cost. For a sequence of point clouds, Fig. 20 shows the CPU times for the setup of the system matrix (left plot), the solution of the arising system with a BiCG scheme (center plot), and the solution with an algebraic multigrid (AMG) scheme (right plot). The latter is performed using SAMG [14] by the Fraunhofer Institute for Algorithms and Scientific Computing.

As expected (Sect. 5.1), the cost of setting up the system matrix is roughly equal for MPS and LSQ method. In fact, MPS is slightly slower with the used simplex method. However, more efficient linear programming methods may turn the tide towards the MPS method. On the other hand, the cost for solving the large linear system is significantly reduced by the MPS method. The speedup factor equals the factor in sparsity, i.e. the MPS approximation does not modify the convergence rate. While the AMG solver shows a cost roughly linear in the number of unknowns, solvers further away from optimal effort (like BiCG) will greatly benefit from the sparsity of the MPS approximation as the number of unknowns increases.

\section{Conclusions and Outlook}

We have presented a meshfree approach that constructs minimal positive stencils for the Laplace operator on a cloud of points. We have shown that under moderate assumptions on the local resolution of the point cloud, positive stencils always exist. The method approximates the Poisson equation by M-matrices, which are optimally sparse. Both properties are beneficial, and they are in general not met by classical least squares approaches. Numer- 
ical tests show that with the presented approach, the approximation error roughly equals the error of classical approaches. The computational cost to construct the new approximation is comparable to the cost of least squares methods. On the other hand, for solving the arising linear system, both cost and memory requirements are reduced significantly due to the optimal sparsity.

An efficient solution of the linear programs is a crucial point in the presented method, worth a deeper analysis. The application to particle methods shall be investigated. While the presented approach can stand as a method of its own, it may also increase the efficiency of other approaches. The minimal stencils can be augmented by additional neighbors and the final stencil be computed by a least squares method. For instance, a local neighborhood radius can be based on the farthest minimal stencil point, thus increasing sparsity and adding local adaptivity to existing meshfree codes.

\section{Acknowledgments}

We would like to thank Axel Klar, Jörg Kuhnert, Sven Krumke, Helmut Neunzert, and Sudarshan Tiwari for helpful discussions and comments. The support by the National Science Foundation is acknowledged. The author was partially supported by NSF grant DMS-0813648.

\section{References}

[1] A. J. Chorin. Numerical solution of the Navier-Stokes equations. Math. Comput., 22:745-762, 1968.

[2] L. Demkowicz, A. Karafiat, and T. Liszka. On some convergence results for FDM with irregular meshes. Comput. Methods Appl. Mech. Engrg., 42:343-355, 1984.

[3] L. Duarte, T. Liszka, and W. Tworzyako. hp-meshless cloud method. Comput. Methods Appl. Mech. Engrg., 139(1-7):263-288, 1996.

[4] L. C. Evans. Partial differential equations, volume 19 of Graduate Studies in Mathematics. American Mathematical Society, 1998.

[5] T. Fujimoto and R. R. Ranade. Characterization of inverse-positive matrices: The Hawkins-Simon condition and the Le Chatelier-Braun principle. Electron. J. Linear Algebra, 11:59-65, 2004. 
[6] J. Fürst and T. Sonar. On meshless collocation approximations of conservation laws: Preliminary investigations on positive schemes and dissipation models. ZAMM Z. Angew. Math. Mech., 81:403-415, 2001.

[7] W. Hackbusch. Iterative Solution of Large Sparse Systems of Equations. Springer, 1994.

[8] J. Kuhnert and S. Tiwari. Finite pointset method based on the projection method for simulations of the incompressible Navier-Stokes equations. In M. Griebel and M. A. Schweitzer, editors, Meshfree methods for Partial Differential Equations, volume 26 of Lecture Notes in Computational Science and Engineering, pages 373-388. Springer, 2002.

[9] P. Lancaster and K. Salkauskas. Surfaces generated by moving least squares methods. Math. Comp., 37:141-158, 1981.

[10] D. Levin. The approximation power of moving least-squares. Math. Comp., 67:1517-1531, 1998.

[11] T. Liszka and J. Orkisz. The finite difference method at arbitrary irregular grids and its application in applied mechanics. Comput. \& Structures, 11:83-95, 1980.

[12] R. A. Lorentz. Multivariate Birkhoff interpolation. Lecture Notes in Mathematics, Springer, 1992.

[13] A. Quarteroni, R. Sacco, and F. Saleri. Numerical mathematics. Springer, 2000.

[14] SCAI SAMG. Webpage at URL: http://www.scai.fhg.de/samg.0.html.

[15] B. Seibold. M-Matrices in meshless finite difference methods. Dissertation, Department of Mathematics, University of Kaiserslautern, 2006.

[16] B. Seibold. Multigrid and M-matrices in the finite pointset method for incompressible flows. In M. Griebel and M. A. Schweitzer, editors, Meshfree methods for Partial Differential Equations III, pages 219-234. Springer, 2007.

[17] B. Seibold. Meshless poisson problems in the finite pointset method: Positive stencils and multigrid. In L.L. Bonilla, M. Moscoso, G. Platero, and J.M. Vega, editors, Progress in Industrial Mathematics at ECMI 2006, Mathematics in Industry, Vol. 12, pages 892-896. Springer, 2008. 
[18] J. R. Shewchuk. Lecture notes on Delaunay mesh generation. University of California at Berkeley, 1999.

[19] R. J. Vanderbei. Linear programming: Foundations and extensions. International Series in Operations Research \& Management Science. Springer, second edition, 2001.

[20] R. S. Varga. Matrix iterative analysis. Springer, second edition, 2000. 